\title{
Charge Transfer in Heavy-Ion Collisions at Relativistic Velocities
}

\author{
D.H. Jakubaßa-Amundsen ${ }^{\star}$ and P.A. Amundsen \\ Institute of Physics, University of Oslo, Norway \\ Received July 17, 1980; revised version September 2, 1980
}

\begin{abstract}
The capture of a target electron into a bound projectile state induced by the electromagnetic field of the fast moving projectile is calculated within the impulse approximation using the impact parameter description. The cross section is shown to decrease with $(\ln E)^{2} / E$ at infinitely high projectile energies $E$.
\end{abstract}

\section{Introduction}

The high-energy treatment of electronic processes is a problem of current interest in atomic collision theory [1]. In the case of $K$-shell ionisation, theoretical investigations were stimulated by experiments carried out with proton beams in the $\mathrm{GeV}$ region [2], and the measured increase of the cross section compared to a nonrelativistic theory could be explained when relativistic effects were included in the calculation.

For rearrangement collisions, in contrary to excitation or ionisation processes, the cross section shows a strong decrease with projectile energy [3], thus making the detection very difficult at high energies. Therefore, the investigation of relativistic effects for charge transfer is more of theoretical interest. Especially the energy dependence of nonradiative electron capture at asymptotically high energies has been an open question. In the present paper we shall concentrate on this process and do not consider the radiative capture which actually is dominating at high velocities [4]. Using a nonrelativistic description, it was shown $[1,3]$ that the second-order term in the Born series falls off with $E^{-11 / 2}$, while the firstorder term behaves as $E^{-6}$, thus indicating the importance of higher-order effects. The impulse approximation is an adequate higher-order theory for charge transfer in fast collisions as it contains no spurious target-projectile overlap terms [3], and leads also to an $E^{-11 / 2}$ behaviour [5] in the nonrelativistic case.

\footnotetext{
* Supported by the Deutsche Forschungsgemeinschaft Present address: Physik-Department T30, Technische Universität München, Reaktorgelände, D-8046 Garching, FRG
}

Alternatively one might consider an eikonal-type approach (cf. Eichler and Chan [6]).

Relativistic calculations have up to now only been carried out in the first-order Born approximation [7] and show an asymptotic decrease with $E^{-1}$ [8]. Actually, there exists a classical relativistic calculation of the second-order term along the lines of the Thomas model $[8,9]$, in which an $E^{-3}$ decrease with energy was found. This would lead to an asymptotic dominance of the first-order term, in contradiction to what is expected for rearrangement collisions. In this paper it will be shown by a consistent relativistic calculation within the impulse approximation, that the dominance of the higher-order terms is retained.

A relativistic description of charge transfer must take into account the appropriate transformation of the projectile field into the target rest frame. This leads to an additional magnetic field which becomes important for high energies, and which is responsible together with the (transformed) electric field, for the increase of the cross section in the case of ionisation $[2,10,11]$. Furthermore, the transformation of the projectile wavefunction into the target system differs from the nonrelativistic case due to the spinor property of the relativistic wavefunctions. As the impulse approximation includes interactions with both the target and the projectile it is not possible, like in a first-order theory, to avoid the transformation of both wavefunctions and interaction field by the choice of a suitable reference frame. These transformation properties are established in Sect. 2. 
Starting from the exact amplitude for charge transfer the impulse approximation is introduced in Sect. 3, together with an additional peaking approximation as in the nonrelativistic case [12].

From this the asymptotic energy dependence of the cross section is derived (Sect. 4).

\section{Relativistic Formulation}

Let us consider the transfer of an electron from the target (charge $Z_{2}$ ) to the projectile (charge $Z_{1}$ ) in systems where $Z_{1} \leqq Z_{2}$. In this case the prior form of the impulse approximation which includes an infinite number of electron-target interactions has to be used [5]. In the semiclassical (impact-parameter) description the transition amplitude for an electron initially bound in a target eigenstate $\psi_{i}(\mathbf{r}, t)$ to a projectile bound state $\psi_{f}\left(\mathbf{r}^{\prime}, t^{\prime}\right)$ is given by the covariant form [13]

$a_{f i}=\frac{1}{i \hbar} \int d t \int d \mathbf{r}\left(\bar{\psi}_{f}^{\prime}\left(\mathbf{r}^{\prime}, t^{\prime}\right) T^{-1}\right)$

$\cdot\left(-e T \mathbb{A}_{P}^{\prime}\left(\mathbf{r}^{\prime}, t^{\prime}\right) T^{-1}\right) \psi_{i}(\mathbf{r}, t)$

where $T$ is a spinor transformation which transforms a wavefunction from the projectile rest system $\left(\mathbf{r}^{\prime}, t^{\prime}\right)$ into the target frame $(\mathbf{r}, t)$ :

$\psi(\mathbf{r}, t)=T \psi^{\prime}\left(\mathbf{r}^{\prime}, t^{\prime}\right)$.

The wavefunction $\bar{\psi}_{f}^{\prime}\left(\mathbf{r}^{\prime}, t^{\prime}\right)=\psi_{f}^{+\prime}\left(\mathbf{r}^{\prime}, t^{\prime}\right) \gamma_{0}$ describes an exact solution of the three-particle problem, i.e., an electron in the field of the projectile and target nuclei. The matrices $\gamma_{0}$ as well as $\gamma^{\mu}$ in the definition of the electromagnetic potential $\mathbb{A}_{P}=\sum_{\mu=0}^{3} \gamma^{\mu} A_{\mu}$ are introduced to demonstrate the invariance of (2.1) under the Lorentz transformation (we use the conventions of [13])

$$
\begin{aligned}
& \left(\begin{array}{l}
c t \\
\mathbf{r}
\end{array}\right)=\Lambda\left(\begin{array}{l}
c t^{\prime} \\
\mathbf{r}^{\prime}
\end{array}\right)+\left(\begin{array}{l}
0 \\
\mathbf{b}
\end{array}\right) ; \\
& \Lambda=\left(\begin{array}{cccc}
\gamma & 0 & 0 & \gamma v / c \\
0 & 1 & 0 & 0 \\
0 & 0 & 1 & 0 \\
\gamma v / c & 0 & 0 & \gamma
\end{array}\right) .
\end{aligned}
$$

$\gamma=\left(1-v^{2} / c^{2}\right)^{-\frac{1}{2}}$ and the $z$-direction is chosen along $\mathbf{v}$. This implies that the projectile moves along a straight line with impact parameter $b$. The projectile field $-e A_{\mu}^{\prime}=V_{P}^{\prime} \delta_{\mu 0}$ is simply given by the Coulomb field $V_{P}^{\prime}=-Z_{1} e^{2} / r^{\prime}$, such that the transformed field $\mathbb{A}_{p}(\mathbf{r}, t)=T \mathbb{A}_{P}^{\prime}\left(\mathbf{r}^{\prime}, t^{\prime}\right) T^{-1}$ can be obtained by means of (2.3):

$$
\begin{aligned}
& V_{P}(\mathbf{r}, t)=-e \gamma_{0} \mathbb{A}_{P}=-e\left(A_{0}-\alpha \mathbf{A}\right) \\
& =-\gamma Z_{1} e^{2} / r^{\prime}\left(1-\alpha_{z} v / c\right)
\end{aligned}
$$

where $\alpha$ are 4-matrices composed of the Pauli spin matrices, for example,

$\alpha_{z}=\left(\begin{array}{cc}0 & \sigma_{z} \\ \sigma_{z} & 0\end{array}\right)$

The transformation matrix $T$ for free particles is given by [13]

$$
T=\left(\frac{1+\gamma}{2}\right)^{\frac{1}{2}}\left(1+\frac{\gamma v / c}{1+\gamma} \alpha_{z}\right)=T^{+}
$$

and the inverse transformation is obtained by means of $T^{-1}=\gamma_{0} T \dot{\gamma}_{0}$ or equivalently $T^{-1}(v)=T(-v)$. As shown in Appendix A, (2.5) describes also the transformation between any solutions of the Dirac equation, provided that the application of (2.5) is followed by the coordinate transformation (2.3). This means that when (2.3)-(2.5) is inserted into (2.1), the transition amplitude is still exact.

\section{Impulse-Peaking Approximation in the Relativistic Case}

In the impulse approximation the exact eigenfunction $\bar{\psi}_{f}$ is replaced by a superposition of target Coulombeigenfunctions $\bar{\psi}_{q, s}$ with momentum $\hbar \mathbf{k}$ weighted with the momentum distribution of the bound projectile state $\psi_{f}$ :

$$
\bar{\psi}_{f}(\mathbf{r}, t)=\sum_{s=1}^{4} \int d \mathbf{q} d \omega_{q}\left(\psi_{f}^{+}(\mathbf{r}, t), q_{s}(\mathbf{r}, t)\right) \bar{\psi}_{q, s}(\mathbf{r}, t) .
$$

Here, $q_{s}$ is a free state of momentum $q$ and energy $\hbar \omega_{q}$ (as given in (3.4)), and $s=1-4$ denotes the spin and particle/antiparticle degrees of freedom. Use has been made of the completeness relation of the spinor amplitudes of $q_{s}, \sum_{s=1}^{4} u_{q}^{+(s)} u_{q}^{(s)}=1$. The brackets (.,.) denote an integration over space and time. The additional energy integral is introduced for convenience and can be carried out by means of the $\delta$-function from the time integral in the overlap term of $\psi_{f}$ and $q_{s}$.

The evaluation of this overlap term can be simplified by using the representation of the projectile state $\psi_{f}$ $=T \psi_{f}^{\prime}$ in the projectile frame and by introducing a complete set of free states $k_{\sigma}$ such that $T$ acts on a free state:

$$
\begin{aligned}
& \left(\psi_{f}^{+\prime}\left(\mathbf{r}^{\prime}, t^{\prime}\right) T, q_{s}(\mathbf{r}, t)\right)=\sum_{\sigma=1}^{4} \int d \mathbf{k} d \omega \\
& \cdot\left(\psi_{f}^{+\prime}\left(\mathbf{r}^{\prime}, t^{\prime}\right), k_{\sigma}^{\prime}\left(\mathbf{r}^{\prime}, t^{\prime}\right)\right)\left(k_{\sigma}^{+\prime}\left(\mathbf{r}^{\prime}, t^{\prime}\right) T, q_{s}(\mathbf{r}, t)\right) .
\end{aligned}
$$


In the impulse approximation the transition amplitude for charge transfer can thus be written as an integral over the product of three terms, the Fourier transform of the final projectile state, the overlap of plane waves in the two different frames, and the ionisation matrix element of the target bound state under the projectile perturbation:

$$
\begin{aligned}
& a_{f i}^{I A}=\frac{1}{i \hbar} \sum_{s=1}^{4} \int d \mathbf{q} d \omega_{q} \sum_{\sigma=1}^{4} \int d \mathbf{k} d \omega\left(\psi_{f}^{+\prime}, k_{\sigma}^{\prime}\right)\left(k_{\sigma}^{+\prime} T, q_{s}\right) \\
& \cdot\left(\bar{\psi}_{q, s},\left(-e \mathbb{A}_{P}\right) \psi_{i}\right) .
\end{aligned}
$$

We proceed with the evaluation of these terms. The free state is given by [14]

$k_{\sigma}^{\prime}\left(\mathbf{r}^{\prime}, t^{\prime}\right)=u_{k}^{(\sigma)}(2 \pi)^{-2} \exp \left(i \mathbf{k} \mathbf{r}^{\prime}-i \omega t^{\prime}\right)$

$u_{k}^{(\sigma)}=\sqrt{\frac{\varepsilon_{k}+m c^{2}}{2 \varepsilon_{k}}}\left(1+\frac{\hbar c \boldsymbol{\alpha} \mathbf{k}(\sigma)}{\varepsilon_{k}+m c^{2}}\right) \mathbf{e}_{\sigma}$

where $\mathbf{k}(\sigma)=\mathbf{k}$ for the particle states $\sigma=1,2$ and $-\mathbf{k}$ for the antiparticle states $\sigma=3,4$. $\mathbf{e}_{\sigma}$ is a 4-dimensional unit vector with the element 1 standing in position $\sigma . \varepsilon_{k}$ is the relativistic energy $\left(m^{2} c^{4}\right.$ $\left.+\hbar^{2} k^{2} c^{2}\right)^{\frac{1}{2}}$. Applying $T$ together with the space-time transformation (2.3) we obtain for the second term

$$
\begin{aligned}
& \int d t d \mathbf{r}\left(T k_{\sigma}^{\prime}\left(\mathbf{r}^{\prime}, t^{\prime}\right)\right)^{+} q_{s}(\mathbf{r}, t) \\
& =\left(u_{k}^{+(\sigma)} T u_{q}^{(s)}\right) e^{i \mathbf{k}_{\perp} \mathbf{b}} \delta\left(\gamma \omega+\gamma v k_{z}-\omega_{q}\right) \\
& \cdot \delta^{2}\left(\mathbf{q}_{\perp}-\mathbf{k}_{\perp}\right) \delta\left(q_{z}-\omega \gamma v / c^{2}-k_{z} \gamma\right) .
\end{aligned}
$$

Thus the integrals over $\mathbf{k}$ and $\omega_{q}$ are trivial. The first term in (3.3) can be written in terms of the Fourier transform $\psi_{f}(\mathbf{k})$ of the projectile state

$$
\begin{aligned}
& \int d t^{\prime} d \mathbf{r}^{\prime} \psi_{f}^{+\prime}\left(\mathbf{r}^{\prime}, t^{\prime}\right) k_{\sigma}^{\prime}\left(\mathbf{r}^{\prime}, t^{\prime}\right) \\
& =(2 \pi)^{\frac{1}{2}} \delta\left(E_{f} / \hbar-\omega\right)\left(\psi_{f}^{+}(\mathbf{k}) u_{k}^{(\sigma)}\right) .
\end{aligned}
$$

When (3.5) and (3.6) are inserted into the transition amplitude (3.3), the integrals over $k, \omega$ and $\omega_{q}$ can be carried out immediately. The only dependence on $\sigma$ is contained in the spinor amplitudes $u_{k}^{(\sigma)}$, such that the spin sum is easily evaluated by means of the completeness relation. Then the transition amplitude reduces to

$$
\begin{aligned}
& a_{f i}^{I A}=\frac{1}{i \hbar \gamma} \sum_{s=1}^{4} \int d \mathbf{q}\left(\psi_{f}^{+}(\mathbf{k}) T u_{q}^{(s)}\right) e^{i \mathbf{q}_{\perp} \mathbf{b}} \\
& \cdot \int d t e^{i / \hbar\left(E_{f} / \gamma+\hbar v q_{z}-E_{i}\right) t} \\
& \left\langle\psi_{q, s}(\mathbf{r})\left|V_{P}\right| \psi_{i}(\mathbf{r})\right\rangle \\
& \mathbf{k}_{\perp}=\mathbf{q}_{\perp}, \quad k_{z}=q_{z} / \gamma-E_{f} v /\left(\hbar c^{2}\right)
\end{aligned}
$$

with $V_{P}$ from (2.4). The energies $E_{f}$ and $E_{i}$ of the projectile and target bound state, respectively, are relativistic energies including the rest mass. In the nonrelativistic limit $(\gamma \rightarrow 1), \mathbf{k} \rightarrow \mathbf{q}-m \mathbf{v} / \hbar$ and the energy phase reduces to $\left(\varepsilon_{f}-\varepsilon_{i}-\frac{1}{2} m v^{2}+\hbar v q_{z}\right) / \hbar$ where $\varepsilon_{f}$ and $\varepsilon_{i}$ are the nonrelativistic energies, thus yielding the same result as obtained in the nonrelativistic theory by means of a Galilean transformation.

The further evaluation proceeds along the lines of the nonrelativistic theory [12]. Using the Fourier representation of the interaction potential

$\frac{1}{r^{\prime}}=\frac{1}{2 \pi^{2}} \int \frac{d \mathbf{s}}{s^{2}} e^{i \mathbf{s}_{\perp}(\mathbf{r}-\mathbf{b})+i s_{\mathrm{z}} \gamma(z-v t)}$

and changing variables in the $\mathbf{q}$ integral to $q_{z}^{\prime}=q_{z} / \gamma$ $-E_{f} v /\left(\hbar c^{2}\right), \mathbf{q}_{\perp}^{\prime}=\mathbf{q}_{\perp}$, we obtain from the time integral in (3.7) the momentum transferred to the electron

$q_{z}^{\prime}=s_{z}-\left(E_{f}-E_{i} / \gamma\right) /(\hbar v) \equiv q_{0}$.

We make use of the fact that the Fourier transform $\psi_{f}\left(\mathbf{q}^{\prime}\right)$ is a rapidly decreasing function of $q^{\prime}$, and take the ionisation matrix element and $u_{q}^{(s)}$ outside the $\mathbf{q}^{\prime}$ integral at the minimum momentum transfer $\mathbf{q}^{\prime}=q_{0} \mathbf{e}_{z}$ given by (3.9). Thus we neglect the transversal components of $\mathbf{q}$ in the ionisation matrix element (and $\left.u_{q}^{(s)}\right)$. In the nonrelativistic case this peaking approximation was found to be quite good in the highvelocity limit [15]. Here it is even more justified because of the large projectile velocity, a large longitudinal momentum $q_{z}$ is required both to reduce the argument of $\psi_{f}$ and to ensure momentum conservation, whereas the transverse components $\mathbf{q}_{\perp}$ can be neglected. By means of this approximation one can carry out the integral over ' $\mathbf{q}$ ' analytically, in addition to further simplifications in the evaluation of the ionisation matrix element. From this one gets

$I^{P}\left(q_{0}\right) \equiv \gamma \int d \mathbf{q}^{\prime} \psi_{f}^{+}\left(\mathbf{q}^{\prime}\right) e^{i \mathbf{q}_{\perp}^{\prime} \mathbf{b}} \int d t e^{i v \gamma\left(q_{z}^{\prime}-q_{0}\right) t}$

$=(2 \pi)^{3 / 2} / v \int d \tau e^{-i q_{0} \tau} \psi_{f}^{+}\left(-\mathbf{R}^{\prime}\right)$

with $\mathbf{R}^{\prime}=\mathbf{b}+\tau \mathbf{e}_{z}$. If a nonrelativistic wavefunction is used (for a light projectile) this expression reduces to

$I^{P}\left(q_{0}\right)=4 \sqrt{2} \pi \frac{b \hat{Z}_{1}^{5 / 2}}{v\left(\hat{Z}_{1}^{2}+q_{0}^{2}\right)^{\frac{1}{2}}} K_{1}\left(b \sqrt{\hat{Z}_{1}^{2}+q_{0}^{2}}\right) \mathbf{e}_{\lambda}^{+}$

$\equiv \hat{I}^{P}\left(q_{0}\right) \mathbf{e}_{\lambda}^{+}$

for a hydrogenic projectile $1 s$ state, where $K_{1}$ is a Macdonald function and $\hat{Z}_{1}=Z_{1} e^{2} m / \hbar . \lambda=1,2$ denotes the two spin degrees of freedom. For the higher projectile states, $\hat{I}^{P}\left(q_{0}\right)$ can be expressed via recursion relations by the functions $K_{1}$ und $K_{0}$.

It is convenient to change variables in (3.8) by means of $s_{z}^{\prime}=\gamma s_{z}, \mathbf{s}_{\perp}^{\prime}=\mathbf{s}_{\perp}$, and we obtain by inserting (3.8)(3.10) into (3.7): 
$a_{f i}^{I A}=-\frac{Z_{1} e^{2}}{2 \pi^{2} i \hbar \gamma} \sum_{s=1}^{4} \int \frac{d \mathbf{s}^{\prime}}{s^{\prime 2}-\left(s_{z}^{\prime} v / c\right)^{2}} e^{-i \mathbf{s}^{\prime} \mathbf{b}}\left(I^{P}\left(q_{0}\right) T u_{q_{z}}^{(s)}\right)$

$\cdot\left\langle\psi_{q_{z}, s}(\mathbf{r})\left|e^{i \mathbf{s}^{\prime} \mathbf{r}}\left(1-\alpha_{z} v / c\right)\right| \psi_{i}(\mathbf{r})\right\rangle$.

This is the relativistic extension of the formula for the charge transfer amplitude from Amundsen and Jakubaßa [12]. As shown in Appendix B, also this expression can be reduced to a two-dimensional integral which has to be carried out numerically. The sum over $s$ includes antiparticle states as intermediate target eigenstates $\psi_{q_{z}, s}$ (originating from the completeness relation of the spinor amplitudes) which will become as important as the particle states when $v \rightarrow c$. One should note that the momentum of $\psi_{q_{z}, s}$ is given by $\hbar q_{z}=\gamma\left(\hbar q_{0}+E_{f} v / c^{2}\right)=-\left(E_{f} / \gamma-E_{i}\right) / v+\hbar s_{z}^{\prime}$ which is centered around $E_{i} / c \approx m c$ for $v \rightarrow c$. This means that the momentum is finite for $\gamma \rightarrow \infty$ and does not increase like $\gamma m v$ as is expected for a relativistic particle moving with velocity $v$. This somewhat paradoxical result is due to the fact that not only the energy of the final state, when seen from the target frame, increases as $\gamma E_{f}$, but also does the width of its momentum distribution, so that the required momentum transfer remains approximately constant. The broadening of the wavefunction in momentum space is, of course, just an effect of the Lorentz-contraction in coordinate space. Consequently one cannot take $k_{z}=0$ in (3.7) as the definition of $q_{z}$ in $\psi_{q_{z}, s}$ which would lead to $q_{z} \approx \gamma m v$, and which was used as an approximation in the nonrelativistic case [5]. Instead it is important to choose the momentum transfer (3.9) as the definition of $q_{z}$ so that this divergence is compensated. For an occupied initial subshell, the total transfer cross section is obtained by means of (3.12)

$\sigma=2 \pi \int_{0}^{\infty} b d b \sum_{i, f}\left|a_{f i}\right|^{2}$

where one has to sum over the electronic initial and final magnetic substates.

\section{High-Energy Limit}

As the projectile energy $E=M_{P} c^{2} \gamma$ is proportional to $\gamma$ the asymptotic behaviour of the transition amplitude is more readily expressed in terms of $\gamma(\gamma \rightarrow \infty)$ than in terms of $v(v \rightarrow c)$. For large $\gamma$, the impulse approximation as well as the peaking approximation should be very good such that the formula (3.12) can be used to study the high-energy behaviour of charge transfer.

Using the fact that $q_{z}$ (and $q_{0}$ ) is finite for $\gamma \rightarrow \infty$ and that $\left(1-\alpha_{z}\right) \psi_{i}(\mathbf{r}) \neq 0$ because the large component dominates in $\psi_{i}$, the ionisation matrix element is independent of $\gamma$ in the limiting case. Also $I^{P}\left(q_{0}\right)$ remains finite as the integral in (3.10) exists and is independent of $\gamma$. The term $T u_{q_{z}}^{(s)}$ increases with $\gamma^{\frac{1}{2}}$ since $T \rightarrow(\gamma / 2)^{\frac{1}{2}}\left(1+\alpha_{z}\right)$.

From this, the behaviour of the $\mathbf{s}^{\prime}$-integral can easily be extracted. Introducing spherical coordinates $s^{\prime}$, $x=\cos \vartheta_{s^{\prime}}, \varphi_{s^{\prime}}$ we have

$\int \frac{d \mathbf{s}^{\prime}}{s^{\prime 2}-\left(s^{\prime} x v / c\right)^{2}}=\int_{0}^{2 \pi} d \varphi_{s^{\prime}} \int_{0}^{\infty} d s^{\prime} \int_{-1}^{1} d x \frac{1}{1-(x v / c)^{2}}$

and thus a further divergence at $v \rightarrow c$ since the remaining integrand is finite at $x= \pm 1$. The contribution from the singularities at $x= \pm c / v$ for $v \rightarrow c$ is $\ln (1-v / c)$ which increases like $\ln \gamma$. The fact that the main contributions come from $x= \pm 1$ where the transverse momentum transfer $\mathbf{s}_{\perp}^{\prime}$ is zero, is indeed consistent with our peaking approximation. Adding up all contributions, we obtain $a_{f i} \sim \gamma^{-\frac{1}{2}} \ln \gamma$. This behaviour is not affected by the integration over impact parameter, such that the total cross section for charge transfer decreases with energy corresponding to

$\sigma \sim(\ln E)^{2} / E, \quad E \rightarrow \infty$.

This logarithmic dependence arises from the behaviour of the Coulomb potential in momentum space. It is not present for a screened (Yukawa) potential. A similar logarithmic asymptotic behaviour is well known for ionisation (see i.e., [10]), and since the impulse approximation, which is well established as a high-energy approximation, contains the same matrix element, the result is not very surprising. It differs from the second-order result obtained by using the relativistic Thomas model $[8,9]$ which decreases with $E^{-3}$, and also from the $1 / E$ behaviour of the Brinkman-Kramers theory. It confirms the nonrelativistic result, that the second-order term asymptotically decreases slower with energy than the first-order term. Actually, this slow decrease with projectile energy becomes only apparent for extremely high energies (in the $\mathrm{GeV}$ region [8]) where the cross section has decreased by many orders of magnitude.

It should finally be remarked that in a real experimental situation the projectile charge will ultimately be screened by the neighbouring atoms of the target. Thus, the $E$-dependence will then change from $(\ln E)^{2} / E$ to $1 / E$ (density effect). At what energies and in which way this change actually will take place, however, is not clear even in the simpler case of ionisation (see i.e., the discussion by Tawara [16]).

To conclude, we have extended the nonrelativistic formulation of the impulse approximation by a con- 
sistent treatment of relativistic effects, including the appropriate transformations of the Dirac spinors and the interaction potential into the target rest frame. As already pointed out by Shakeshaft [8] the inclusion of relativistic effects leads to an increase of the cross section. A rough semirelativistic estimate following the lines of this paper suggests a ten percent correction to the nonrelativistic result at energies of the order of $100 \mathrm{MeV}$, where the cross section is already far beyond experimental detection. In evaluating the relativistic impulse approximation at high energies, a behaviour of $(\ln E)^{2} / E$ was found for the capture cross section. Although this result differs from the nonrelativistic estimate, it stresses again the importance of higher-order effects in the theory of charge transfer.

We should like to thank E. Eriksen for a clarifying discussion.

\section{Appendix A}

We prove that the matrix $T$ in (2.5) describes the correct transformation of any solution of the Dirac equation from the projetile frame to the target frame by showing that the transformed wavefunction is a solution of the transformed Dirac equation.

Let $\psi^{\prime}\left(\mathbf{r}^{\prime}, t^{\prime}\right)$ be a solution of the Dirac equation in the projectile frame

$$
i \hbar \frac{\partial \psi^{\prime}\left(\mathbf{r}^{\prime}, t^{\prime}\right)}{\partial t^{\prime}}=\left(\frac{\hbar}{i} c \alpha \nabla^{\prime}+\beta m c^{2}-\frac{Z_{1} e^{2}}{r^{\prime}}\right) \psi^{\prime}\left(\mathbf{r}^{\prime}, t^{\prime}\right)
$$

where for simplicity we consider only the action of a Coulomb field on the electron. Then we claim that the transformed function

$\psi(\mathbf{r}, t)=\left(\frac{1+\gamma}{2}\right)^{\frac{1}{2}}\left(1+\frac{\gamma v / c}{1+\gamma} \alpha_{z}\right) \psi^{\prime}\left(\mathbf{r}^{\prime}, t^{\prime}\right)$

is a solution of the Dirac equation in the target frame:

$i \hbar \frac{\partial \psi(\mathbf{r}, t)}{\partial t}=\left(\frac{\hbar}{i} c \alpha \boldsymbol{\nabla}+\beta m c^{2}-\gamma \frac{Z_{1} e^{2}}{r^{\prime}}\left(1-\alpha_{z} v / c\right)\right) \psi(\mathbf{r}, t)$

To this aim we apply the space-time transformation (2.3) to (A.3). Using $\partial / \partial t=\gamma \partial \partial \partial t^{\prime}-\gamma v \nabla_{z^{\prime}}$ and $\nabla_{z}=$ $-\gamma\left(v / c^{2}\right) \partial / \partial t^{\prime}+\gamma \nabla_{z^{\prime}}$, (A.3) turns into

$$
\begin{aligned}
& i \hbar \gamma\left(1-\alpha_{z} v / c\right) \frac{\partial \psi(\mathbf{r}, t)}{\partial t^{\prime}} \\
& =\left(\frac{\hbar}{i} c \alpha_{\perp} \nabla_{\perp}^{\prime}+\frac{\hbar}{i} \gamma c\left(\alpha_{z}-v / c\right) \nabla_{z^{\prime}}+\beta m c^{2}\right. \\
& \left.-\gamma \frac{Z_{1} e^{2}}{r^{\prime}}\left(1-\alpha_{z} v / c\right)\right) \psi(\mathbf{r}, t) .
\end{aligned}
$$

Inserting (A.2) for $\psi(\mathbf{r}, t)$ and expressing the derivative $\partial \psi^{\prime}\left(\mathbf{r}^{\prime}, t^{\prime}\right) / \partial t^{\prime}$ by means of (A.1) it follows after a short calculation that (A.4) reduces to an identity, which proves our statement.

\section{Appendix B}

In this appendix we evaluate the transfer amplitude (3.12). We confine ourselves to a light projectile and describe the projectile state $\psi_{f}^{\prime}$ by a nonrelativistic function $\varphi_{f}^{\prime} \mathbf{e}_{\lambda}(\lambda=1,2)$. The initial state $\psi_{i}$, however, must be described by a relativistic function even for small $Z_{2}$, since it enters the matrix element for the transition to a target state $\psi_{q_{z}, s}$ which is characterised by the relativistic velocity $v$. Orthogonality between initial and final states thus requires both functions, $\psi_{q_{z}, s}$ and $\psi_{i}$, to be relativistic. The relativistic Coulomb wave $\psi_{q_{z}, s}$ does not exist in closed form, and one has to describe it by means of projection on angular momentum eigenstates $\kappa_{j_{1} l_{1} m_{1}}$ of momentum $\kappa$. To this aim we introduce the complete set

$\sum_{j_{1} l_{1} m_{1}} \int_{0}^{\infty} d \kappa\left|\kappa_{j_{1} l_{1} m_{1}}\right\rangle\left\langle\kappa_{j_{1} l_{1} m_{1}}\right|=1$

such that

$\left\langle\psi_{q_{z}, s}\right|=\int_{0}^{\infty} d \kappa \sum_{j_{1} l_{1} m_{1}}\left\langle q_{z, s} \mid \kappa_{j_{1} l_{1} m_{1}}\right\rangle\left\langle\psi_{j_{1} l_{1} m_{1}}^{\kappa}\right|$

where $\psi_{j_{1} l_{1} m_{1}}^{\kappa}$ is a target eigenstate of angular momentum $j_{1}, l_{1}$ and magnetic quantum number $m_{1}$. The momentum-normalised free state $\kappa_{j_{1} l_{1} m_{1}}$ is given by [14]

$$
\begin{aligned}
& \kappa_{j_{1} l_{1} m_{1}}(\mathbf{r})=\kappa(2 / \pi)^{\frac{1}{2}} \\
& \left(\begin{array}{l}
\sqrt{\frac{\varepsilon_{\kappa}+m c^{2}}{2 \varepsilon_{\kappa}}} i^{l_{1}} j_{l_{1}}(\kappa r) Y_{j_{1} l_{1} m_{1}}(\Omega) \\
-\sqrt{\frac{\varepsilon_{\kappa}-m c^{2}}{2 \varepsilon_{\kappa}}} i^{l_{1}} j_{l_{1}}(\kappa r) Y_{j_{1} l_{1}^{\prime} m_{1}}(\Omega)
\end{array}\right)
\end{aligned}
$$

with $l_{1}^{\prime}=2 j_{1}-l_{1} . Y_{j l m}(\Omega)$ is a spherical harmonic spinor.

When inserting the expansion (B.1) into (3.12) the sum over $s$ can be carried out by means of the completeness relation

$$
\begin{aligned}
& \sum_{s=1}^{4}\left(\mathbf{e}_{\lambda}^{+} T u_{q_{z}}^{(s)}\right) \int d \mathbf{r} u_{q_{z}}^{+(s)} e^{-i q_{z} z} \kappa_{j_{1} l_{1} m_{1}}(\mathbf{r}) \\
& =\mathbf{e}_{\lambda}^{+} T \int d \mathbf{r} e^{-i q_{z} z} \kappa_{j_{1} l_{1} m_{1}}(\mathbf{r})
\end{aligned}
$$

and the overlap integral is easily evaluated if one uses the partial wave expansion of $\exp \left(-i q_{z} z\right)$ such that the angular integration can be performed im- 
mediately. One finds with the help of the orthogonality relation of the spherical Bessel functions

$$
\begin{aligned}
& \int_{0}^{\infty} r^{2} d r j_{\lambda}(q r) j_{\lambda}\left(q^{\prime} r\right)=\pi /\left(2 q^{2}\right) \delta\left(q-q^{\prime}\right): \\
& \mathbf{e}_{\lambda}^{+} T \int d \mathbf{r} e^{-i q_{z} z} \kappa_{j_{1} l_{1} m_{1}}(\mathbf{r}) \\
& ==\delta\left(\left|q_{z}\right|-\kappa\right) P^{\lambda}\left(\kappa, j_{1} l_{1} m_{1}\right) P^{2}\left(\kappa, j_{1} l_{1} m_{1}\right)=\frac{(1+\gamma)^{\frac{\lambda}{2}}}{4 \pi \kappa} \\
& \left(\delta_{m_{1}, \frac{1}{2}} \delta_{\lambda, 1}+(-1)^{l_{1}+\frac{1}{2}-j_{1}} \delta_{m_{1},-\frac{1}{2}} \delta_{\lambda, 2}\right) \\
& \cdot\left[\left(2 l_{1}+1\right)^{\frac{1}{2}} \sqrt{\frac{\varepsilon_{\kappa}+m c^{2}}{2 \varepsilon_{\kappa}}}\left(\frac{q_{z}}{\kappa}\right)^{l_{1}}\left(l_{1} \frac{1}{2} j_{1} \mid 0 \frac{1}{2} \frac{1}{2}\right)\right. \\
& \left.-\frac{\gamma v / c}{1+\gamma}\left(2 l_{1}^{\prime}+1\right)^{\frac{1}{2}} \sqrt{\frac{\varepsilon_{\kappa}-m c^{2}}{2 \varepsilon_{\kappa}}}\left(\frac{q_{z}}{\kappa}\right)^{l_{1}^{\prime}}\left(l_{1} \frac{1}{2} j_{1} \mid 0 \frac{1}{2} \frac{1}{2}\right)\right]
\end{aligned}
$$

where the brackets denote Clebsch-Gordan coefficients. This overlap integral is only nonvanishing if $\left|m_{1}\right|=\frac{1}{2}$ which can be traced back to the fact that the momentum of $q_{z, s}(\mathbf{r})$ lies in the $z$-direction, due to the peaking approximation.

It remains to evaluate the matrix element

$M \equiv\left\langle\psi_{j_{1} l_{1} m_{1}}^{\kappa}(\mathbf{r})\left|e^{i \mathbf{s}^{\prime} \mathbf{r}}\left(1-\alpha_{z} v / c\right)\right| \psi_{i}(\mathbf{r})\right\rangle$.

As the target eigenstates $\psi(\mathbf{r})$ are given in the angular momentum representation [14]

$\psi(\mathbf{r})=\left(\begin{array}{ll}f_{j l}(r) & Y_{j l m}(\Omega) \\ i g_{j l}(r) & Y_{j l^{\prime} m}(\Omega)\end{array}\right)$

it is again convenient to make a multipole expansion of $\exp \left(i \mathbf{s}^{\prime} \mathbf{r}\right)$ for the sake of the angular integration. Characterising $\psi_{i}(\mathbf{r})$ by $j_{2}, l_{2}$ and $m_{2}$ and making use of the fact that $\alpha_{z}$ mixes the large and small components of $\psi$,

$\left(\begin{array}{cc}0 & \sigma_{z} \\ \sigma_{z} & 0\end{array}\right) \psi_{i}(\mathbf{r})=\left(\begin{array}{ccc}i g_{j_{2} l_{2}}(r) & \sigma_{z} Y_{j_{2} l_{2}^{\prime} m_{2}}(\Omega) \\ f_{j_{2} l_{2}}(r) & \sigma_{z} Y_{j_{2} l_{2} m_{2}}(\Omega)\end{array}\right)$

one obtains

$$
\begin{aligned}
& M=4 \pi \sum_{l m} i^{l} Y_{l m}^{*}\left(\Omega_{s^{\prime}}\right) M_{j l m}\left(s^{\prime}, \kappa\right) \\
& M_{j l m}\left(s^{\prime}, \kappa\right)=F_{l}^{(1)} W_{j l m}+i v / c \\
& \cdot\left(F_{l}^{(2)} A_{j l m}\left(l_{1}^{\prime}, l_{2}\right)-F_{l}^{(3)} A_{j l m}\left(l_{1}, l_{2}^{\prime}\right)\right) .
\end{aligned}
$$

$F_{l}^{(i)}$ denote the radial integrals

$$
\begin{aligned}
& F_{l}^{(1)}=\int_{0}^{\infty} r^{2} d r j_{l}\left(s^{\prime} r\right) \\
& \cdot\left[f_{j_{1} l_{1}}^{*}(r) f_{j_{2} l_{2}}(r)+g_{j_{1} l_{1}}^{*}(r) g_{j_{2} l_{2}}(r)\right] \\
& F_{i}^{(2)}=\int_{0}^{\infty} r^{2} d r j_{l}\left(s^{\prime} r\right) g_{j_{1} l_{1}}^{*}(r) f_{j_{2} l_{2}}(r) \\
& F_{l}^{(3)}=\int_{0}^{\infty} r^{2} d r j_{l}\left(s^{\prime} r\right) f_{j_{1} l_{1}}^{*}(r) g_{j_{2} l_{2}}(r)
\end{aligned}
$$

and the coefficients $W_{j l m}$ and $A_{j l m}\left(l_{1}, l_{2}\right)$ are the angular integrals

$$
\begin{aligned}
& W_{j l m}=\int d \Omega Y_{j_{1} l_{1} m_{1}}^{*}(\Omega) Y_{l m}(\Omega) Y_{j_{2} l_{2} m_{2}}(\Omega) \\
& =(-1)^{\frac{1}{2}+m_{1}}(4 \pi)^{-\frac{1}{2}} \hat{j_{1}} \hat{j_{2}} \hat{l}\left(\begin{array}{ccc}
j_{2} & l & j_{1} \\
-m_{2} & -m & m_{1}
\end{array}\right)\left(\begin{array}{ccc}
j_{2} & l & j_{1} \\
\frac{1}{2} & 0 & -\frac{1}{2}
\end{array}\right) \\
& A_{j l m}\left(l_{1}, l_{2}\right)=\int d \Omega Y_{j_{1} l_{1} m_{1}}^{*}(\Omega) Y_{l m}(\Omega) \sigma_{z} Y_{j_{2} l_{2} m_{2}}(\Omega) \\
& =(-1)^{m_{2}-l-\frac{1}{2}}(4 \pi)^{-\frac{1}{2}} \hat{j}_{1} \hat{j_{2}} \hat{l} \hat{l}_{1} \hat{l}_{2}\left(\begin{array}{ccc}
l_{1} & l_{2} & l \\
0 & 0 & 0
\end{array}\right) \\
& \cdot \sum_{\mu}\left(\begin{array}{ccc}
l & l_{2} & l_{1} \\
m & \mu & -m-\mu
\end{array}\right)\left(\begin{array}{ccc}
\frac{1}{2} & j_{1} & l_{1} \\
m_{2}-\mu & -m_{1} & m+\mu
\end{array}\right) \\
& \cdot\left(\begin{array}{ccc}
l_{2} & \frac{1}{2} & j_{2} \\
\mu & m_{2}-\mu & -m_{2}
\end{array}\right)
\end{aligned}
$$

if $l_{1}+l_{2}+l$ is even, and they vanish otherwise. The brackets denote Wigner $3 j$ symbols and $\hat{j}=(2 j+1)^{\frac{1}{2}}$. Because of this parity selection rule either $W_{j l m}$ vanishes for a given $l$, or $A_{j l m}\left(l_{1}^{\prime}, l_{2}\right)$ and $A_{j l m}\left(l_{1}, l_{2}^{\prime}\right)$ because $l_{1,2}^{\prime}=l_{1,2} \pm 1$. However, as the transition amplitude contains a sum over both $l$ and $l_{1}$, the contributions of the electric field (proportional to $W_{j l m}$ ) and of the magnetic field (proportional to $A_{j l m}$ ) add coherently.

The radial integrals can be evaluated analytically for hydrogenic wave functions [17]. For a $1 s_{\frac{1}{2}}$ initial state, they are expressed by the real and imaginary part of

$$
\begin{aligned}
& I_{0}=\int_{0}^{\infty} d r r^{\gamma_{1}+\gamma_{2}} j_{l}\left(s^{\prime} r\right) e^{-\alpha_{0} r} e_{1}^{i \kappa r} F_{1}\left(\gamma_{1}-i v, 2 \gamma_{1}+1,\right. \\
& -2 i \kappa r) \\
& =\frac{\pi^{\frac{1}{2}}}{2 \Gamma(l+3 / 2)}\left(\frac{s^{\prime}}{2}\right)^{l} \frac{\Gamma\left(\gamma_{1}+\gamma_{2}+l+1\right)}{\left(\alpha_{0}+i\left(s^{\prime}-\kappa\right)\right)^{\gamma_{1}+\gamma_{2}+l+1}} \\
& \cdot F_{2}\left(\gamma_{1}+\gamma_{2}+l+1 ; l+1, \gamma_{1}-i v ; 2 l+2,2 \gamma_{1}+1\right. \\
& \left.\frac{2 i s^{\prime}}{\alpha_{0}+i\left(s^{\prime}-\kappa\right)},-\frac{2 i \kappa}{\alpha_{0}+i\left(s^{\prime}-\kappa\right)}\right)
\end{aligned}
$$

where

$$
\begin{aligned}
& \gamma_{i}=\sqrt{\left(i_{i}+\frac{1}{2}\right)^{2}-\left(Z_{2} e^{2} / \hbar c\right)^{2}}, \\
& \alpha_{0}=Z_{2} e^{2} m / \hbar^{2}, \quad v=Z_{2} e^{2} \varepsilon_{\kappa} /\left(\hbar^{2} c^{2} \kappa\right)
\end{aligned}
$$

and ${ }_{1} F_{1}$ and $F_{2}$ are confluent and Appell hypergeometric functions, respectively. $F_{2}$ can be further reduced to a finite sum of hypergeometric functions ${ }_{2} F_{1}[18]$. One obtains

$$
\begin{aligned}
& F_{l}^{(1)}=N_{0}\left(\sqrt{1+m c^{2} / \varepsilon_{\kappa}} \sqrt{1+\gamma_{2}} \operatorname{Im}\left\{e^{i \xi} I_{0}\right\}\right. \\
& \left.-\sqrt{1-m c^{2} / \varepsilon_{\kappa}} \sqrt{1-\gamma_{2}} \operatorname{Re}\left\{e^{i \xi} I_{0}\right\}\right) \\
& F_{l}^{(2)}=N_{0} \sqrt{1-m c^{2} / \varepsilon_{\kappa}} \sqrt{1+\gamma_{2}} \operatorname{Re}\left\{e^{i \xi} I_{0}\right\} \\
& F_{i}^{(3)}=-N_{0} \sqrt{1+m c^{2} / \varepsilon_{\kappa}} \sqrt{1-\gamma_{2}} \operatorname{Im}\left\{e^{i \xi} I_{0}\right\}
\end{aligned}
$$


with

$N_{0}=(-i)^{l_{1}}(2 \pi)^{-\frac{1}{2}}\left(2 \alpha_{0}\right)^{\gamma_{2}+\frac{1}{2}}(2 \kappa)^{\gamma_{1}} e^{\pi v / 2}$

$\frac{\left|\Gamma\left(\gamma_{1}+1+i v\right)\right|}{\sqrt{\Gamma\left(2 \gamma_{2}+1\right)} \Gamma\left(2 \gamma_{1}+1\right)}$

and

$e^{2 i \xi}=\frac{\mp\left(j_{1}+\frac{1}{2}\right)-i v m c^{2} / \varepsilon_{\kappa}}{\gamma_{1}-i v} \quad$ for $j_{1}=l_{1} \pm \frac{1}{2}$

We introduce spherical coordinates in the remaining $\mathbf{s}^{\prime}$ integral in the transition amplitude (3.12), according to (4.1). Then, due to the peaking approximation, the integral over the angle $\varphi_{s^{\prime}}$ can be carried out analytically since the matrix element $M_{j l m}\left(s^{\prime}, \kappa\right)$ in (B.8) is independent of $\varphi_{s^{\prime}}$ :

$$
\begin{aligned}
& \int_{0}^{2 \pi} d \varphi_{s^{\prime}} e^{-i s^{\prime} b \sqrt{1-x^{2}} \cos \varphi_{s^{\prime}}} Y_{l m}^{*}\left(\Omega_{s^{\prime}}\right) \\
& =(-i)^{|m|} \sqrt{\pi(2 l+1) \frac{(l-m) !}{(l+m) !}} P_{l}^{m}(x) J_{|m|}\left(s^{\prime} b \sqrt{1-x^{2}}\right)
\end{aligned}
$$

where $P_{l}^{m}$ is a Legendre polynomial and $J_{|\boldsymbol{}| \boldsymbol{m} \mid}$ a Bessel function. Thus the calculation of the transition amplitude (3.12) reduces to the numerical evaluation of a two-dimensional integral:

$a_{f i}^{I A}=-\frac{2 Z_{1} e^{2}}{i \sqrt{\pi} h \gamma} \int_{0}^{\infty} d s^{\prime} \int_{-1}^{1} d x \frac{1}{1-(x v / c)^{2}} \hat{I}^{P}\left(q_{0}\right)$

$\cdot \sum_{j_{1} l_{1}} \sum_{m_{1}= \pm \frac{1}{2}} P^{2}\left(\left|q_{z}\right|, j_{1} l_{1} m_{1}\right) \sum_{l m} i^{l-|m|}$

$\cdot \sqrt{(2 l+1) \frac{(l-m) !}{(l+m) !}} P_{l}^{m}(x) J_{|m|}\left(s^{\prime} b \sqrt{1-x^{2}}\right) M_{j l m}\left(s^{\prime}, \mid q_{z}\right)$.
When carrying out the sum over the angular momenta, one has to keep in mind the selection rules from the angular integrals (B.10) and that, due to the large momentum transfer for high $v$, only the lowest $l$ values contribute appreciably.

\section{References}

1. Dettmann, K.: Springer tracts in modern physics. Vol. 58, p. 119. Berlin, Heidelberg, New York: Springer 1971

2. Anholt, R., Nagamiya, S., Rasmussen, J.O., Bowman, H., Ioannou-Yannou, J.G., Rauscher, E.: Phys. Rev. A 14, 2103 (1976)

3. McDowell, M.R.C., Coleman, J.P.: Introduction to the theory of ion-atom collisions. Amsterdam: North-Holland 1970

4. Raisbeck, G., Yiou, F.: Phys. Rev. A4, 1858 (1971)

5. Briggs, J.S.: J. Phys. B 10, 3075 (1977)

6. Eichler, J., Chan, F.T.: Phys. Rev. A 20, 104 (1979)

7. Mittleman, M.H.: Proc. Phys. Soc. 84, 453 (1964)

8. Shakeshaft, R.: Phys. Rev. A20, 779 (1979)

9. Moiseiwitsch, B.L., Stockman, S.G.: J. Phys. B 12, L 695 (1979)

10. Davidovic, D.M., Moiseiwitsch, B.L., Norrington, P.H.: J. Phys. B 11, 847 (1978)

11. Aashamar, K., Amundsen, P.A.: (submitted for publication)

12. Amundsen, P.A., Jakubaßa, D.H.: J. Phys. B13, L467 (1980)

13. Bjorken, D, Drell, S.D.: Relativistische Quantenmechanik. Mannheim: BI 1964

14. Achieser, A.I., Berestezki, W.B.: Quantenelektrodynamik. Frankfurt: Deutsch 1962

15. Jakubaßa-Amundsen, D.H., Amundsen, P.A.: Z. Phys. A297, $203(1980)$

16. Tawara, H.: Invited Papers and Progress Reports, $X^{\text {th }}$ ICPEAC. Paris. Watel, G. (ed.), p. 311. Amsterdam: North Holland 1977

17. Jamnik, D., Zupanćić, Ć. Danske Vidensk. Selsk., Mat.-Fys. Meddr. 31, 1 (1957)

18. Amundsen, P.A.: J. Phys. B 11, 3197 (1978)

D.H. Jakubaßa-Amundsen

P.A. Amundsen

Institute of Physics

University of Oslo

Box 1048 Blindern

Oslo 3, Norway 